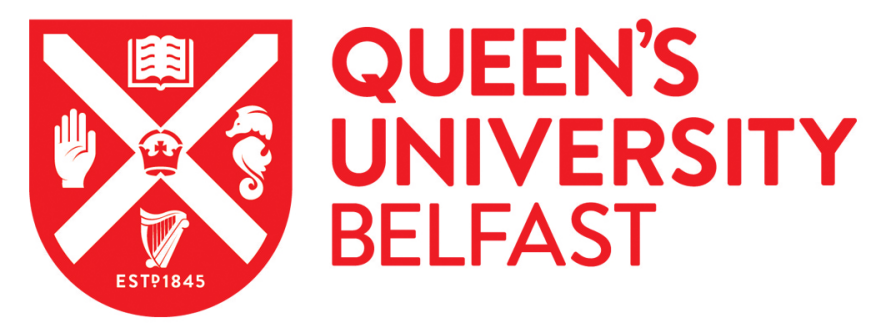

\title{
Ex Vivo Expansion of Human Outgrowth Endothelial Cells Leads to IL- 8-Mediated Replicative Senescence and Impaired Vasoreparative
} Function

Medina, R. J., O'Neill, C. L., O'Doherty, T. M., Chambers, S. E. J., Guduric-Fuchs, J., Neisen, J., Waugh, D. J., Simpson, D. A., \& Stitt, A. W. (2013). Ex Vivo Expansion of Human Outgrowth Endothelial Cells Leads to IL-8Mediated Replicative Senescence and Impaired Vasoreparative Function. Stem Cells, 31(8), 1657-1668. https://doi.org/10.1002/stem.1414

Published in:

Stem Cells

Document Version:

Publisher's PDF, also known as Version of record

Queen's University Belfast - Research Portal:

Link to publication record in Queen's University Belfast Research Portal

Publisher rights

Copyright $\odot 2013$ AlphaMed Press

Open Access

General rights

Copyright for the publications made accessible via the Queen's University Belfast Research Portal is retained by the author(s) and / or other copyright owners and it is a condition of accessing these publications that users recognise and abide by the legal requirements associated with these rights.

Take down policy

The Research Portal is Queen's institutional repository that provides access to Queen's research output. Every effort has been made to ensure that content in the Research Portal does not infringe any person's rights, or applicable UK laws. If you discover content in the

Research Portal that you believe breaches copyright or violates any law, please contact openaccess@qub.ac.uk. 


\title{
Stem Celis
}

\section{Tissue-Specific Stem Cells}

\section{Ex Vivo Expansion of Human Outgrowth Endothelial Cells Leads to IL-8-Mediated Replicative Senescence and Impaired Vasoreparative Function}

\author{
Reinhold J. Medina, ${ }^{\text {a }}$ Christina L. O’Neill, ${ }^{a}$ T. Michelle O'Doherty, ${ }^{a}$ Sarah E.J. Chambers, ${ }^{\text {a }}$ \\ Jasenka Guduric-Fuchs, ${ }^{a}$ Jessica Neisen, ${ }^{b}$ David J. Waugh, ${ }^{b}$ David A. Simpson, ${ }^{\text {a }}$ Alan W. Stitt ${ }^{\text {a }}$ \\ ${ }^{\mathrm{a}}$ Centre for Vision and Vascular Science and ${ }^{\mathrm{b}}$ Centre for Cancer Research and Cell Biology, School of Medicine, \\ Dentistry, and Biomedical Science, Queen's University Belfast, Belfast, United Kingdom
}

Key Words. Angiogenesis • Cell therapy • Endothelial progenitor • IL8 • Senescence

\begin{abstract}
Harnessing outgrowth endothelial cells (OECs) for vasoreparative therapy and tissue engineering requires efficient ex vivo expansion. How such expansion impacts on OEC function is largely unknown. In this study, we show that OECs become permanently cell-cycle arrested after ex vivo expansion, which is associated with enlarged cell size, $\beta$-galactosidase activity, DNA damage, tumor suppressor pathway activation, and significant transcriptome changes. These senescence hallmarks were coupled with low telomerase activity and telomere shortening, indicating replicative senescence. OEC senescence limited their regenerative potential by impairing vasoreparative properties in vitro and in vivo. Integrated transcriptome-
\end{abstract}

proteome analysis identified inflammatory signaling pathways as major mechanistic components of the OEC senescence program. In particular, IL8 was an important facilitator of this senescence; depletion of IL8 in OECs significantly extended ex vivo lifespan, delayed replicative senescence, and enhanced function. While the ability to expand OEC numbers prior to autologous or allogeneic therapy remains a useful property, their replicative senescence and associated impairment of vasorepair needs to be considered. This study also suggests that modulation of the senescence-associated secretory phenotype could be used to optimize OEC therapy. STEM Cells 2013;31:16571668

Disclosure of potential conflicts of interest is found at the end of this article.

\section{INTRODUCTION}

Harnessing the vasoreparative potential of endothelial progenitor cells (EPCs) has emerged as a viable therapeutic option for ischemic diseases such as myocardial infarction [1], peripheral arterial disease [2], and ischemic retinopathies [3]. In addition, generation of new blood vessels is a critical step in tissue engineering strategies, and EPCs are currently being considered for enhancing wound repair, osteogenesis, and development of 3D-vascularized liver organoids. The therapeutic potential of EPCs underpins the necessity to develop an efficient, clinically relevant EPC isolation and ex vivo expansion protocol for subsequent use in therapeutic angiogenesis and tissue engineering.

A frequently overlooked hurdle in adult stem cell therapies is the generation of sufficient cell numbers required for delivery into patients [4] and viability of an EPC-based cytotherapy relies on safe and efficient expansion of cell num- bers ex vivo from tens to millions. Large-scale expansion of adult stem cells is often limited by the exhaustion of their replicative potential. Although many reports highlight the remarkable proliferative capacity of a distinct EPC subtype known as outgrowth endothelial cells (OECs) or endothelial colony forming cells (ECFCs) [5, 6], the implications for their long-term expansion remain largely unknown and detailed molecular and functional evaluation is required.

Cellular senescence has been reported to occur during ex vivo expansion of adult stem cells [7]. Senescence of mesenchymal stem cells and hematopoietic stem cells (HSCs) is associated with significant changes in their differentiation potential and gene expression profile $[8,9]$ which impact on their therapeutic efficacy. In this study, we have investigated the ex vivo growth dynamics and senescence program of this well-defined and characterized EPC subpopulation commonly known as OECs $[10,11]$ or ECFCs $[5,6]$. We show that OECs can be successfully expanded ex vivo, although these cells ultimately undergo senescence upon exhaustion of their

Author contributions: R.J.M. and A.W.S.: conception and design; R.J.M., C.L.O., T.M.O., S.E.C., and J.G-F.: collection and/or assembly of data; R.J.M., C.L.O., J.G-F., and A.W.S.: data analysis and interpretation; J.N.: performed IL8 quantification by ELISA; D.J.W. and D.A.S.: provided intellectual input; R.J.M. and A.W.S.: manuscript writing. All authors have read and approved the manuscript.

Correspondence: Alan W. Stitt, Ph.D., Centre for Vision \& Vascular Science, School of Medicine, Dentistry \& Biomedical Science, Queen's University Belfast, Belfast BT12 6BA, U.K. Telephone: +44-28-90632546; Fax: +44-28-90632699; e-mail: a.stitt@qub.ac. uk Received August 21, 2012; Revised March 13, 2013; accepted for publication March 18, 2013; first published online in STEM CELLS EXPRESS April 30, 2013. (C) AlphaMed Press 1066-5099/2013/\$30.00/0 doi: 10.1002/stem.1414 
replicative potential. In addition, we demonstrate that in comparison to presenescent (early-passage) OECs, senescent OECs are dysfunctional, and by analysis of their transcriptome, proteome, and secretome, we have identified molecular players during OEC senescence. interleukin 8 (IL8) was recognized as a critical component and here we report that by knocking down expression of this cytokine in OECs, the establishment of cellular senescence was significantly delayed. Through better understanding of the molecular pathways that control OEC senescence, we may ultimately be able to overcome the major challenge of efficiently expanding cell numbers without diminishing functionality.

\section{Materials ANd Methods}

\section{Cell Isolation and Culture}

This study was approved by the Office for Research Ethics Committees Northern Ireland. OECs were isolated from human peripheral (PB) and umbilical cord blood (CB) as previously described [11]. Human PB was obtained with full ethical approval from healthy, nonsmoking volunteers aged 2235 years who were not receiving any medication. Umbilical $\mathrm{CB}$ was obtained with full ethical approval from full-term neonates. Operational definition for early-passage OECs in this study was cells that were in their exponential phase of the growth curve (between passage 4 and 6 for PB-derived OECs [PB-OECs] and between passage 8 and 12 for umbilical CB-derived OECs [CB-OECs]), while late-passage OECs were cells that have reached their Hayflick limit. The CASY cell counter-analyzer (Roche, Germany, http://www.rocheapplied-science.com) was used for cell counts and cellular size assessment.

\section{Cytochemical Staining}

Senescence-associated $\beta$-galactosidase (SA- $\beta$-Gal), bromodeoxyuridine (BrdU) incorporation, and $\gamma \mathrm{H} 2 \mathrm{AX}$ inmunocytochemistry assays were performed using standard protocols which are outlined in Supporting Information.

\section{Cell Cycle Analysis}

Detailed in Supporting Information.

\section{Telomere Length and Telomerase Activity Assays}

The telomere PNA kit/fluorescein isothiocyanate (FITC) for flow cytometry (DAKO, http://www.dako.com) was used to measure telomere lengths according to manufacturer's protocol. For measurement of telomerase activity, the quantitative telomerase detection kit (US Biomax, http://www.biomax.us) was used in accordance with manufacturer's guidelines.

\section{RNA Extraction and Microarray Analysis}

Total RNA was extracted using an RNAqueous kit (Ambion Life Technologies, http://www.ambion.com), and $1 \mu \mathrm{g}$ of RNA from each cell sample was labeled and hybridized to an Illumina WG-6 v3.0 Expression Beadchip (Illumina, http:// www.illumina.com). Samples analyzed in the array were three PB-OEC biological replicates of early and late passages. Gene expression data obtained from Illumina Beadstudio was normalized by using an "R" bioconductor with a "lumi" package. Data were processed and analyzed using various bioinformatics software packages detailed in Supporting Information. Microarray data are available at the ARRAYEXPRESS database with accession number E-MTAB-1388.

\section{Real-Time Reverse-Transcription Polymerase Chain Reaction}

Detailed in Supporting Information.

\section{Human Angiogenesis and Cytokine Protein Arrays}

OEC protein lysates and conditioned media were analyzed by using the Proteome Profiler human angiogenesis and cytokine arrays (R\&D Systems, Minneapolis, MN, http://www.rndsystems.com) in accordance with manufacturer guidelines. Spots were detected by using chemiluminescence, and densitometry was quantified using Image J software.

\section{IL8 ELISA}

IL8 levels were assessed by ELISA using Human IL-8 ELISA MAX (Biolegend, http://www.biolegend.com) according to the manufacturer's instructions.

\section{In Vitro Cell Functional Assays}

Tubulogenesis, migration, and clonogenic assays were performed following standard protocols detailed in Supporting Information.

\section{Murine Model of Retinal Ischemia}

C57BL/6 mice were purchased from Harlan Laboratories, U.K. All experiments were performed in compliance with the Association for Research in Vision and Ophthalmology statement for the use of animals in Ophthalmic and Vision Research and UK Home Office regulations. Ischemic retinopathy was induced in C57BL/6 mice by using the previously described oxygen-induced retinopathy model (OIR) [11] and summarized in Supporting Information.

\section{IL8 Small Hairpin RNA (shRNA) Lentiviral Transduction}

IL8 gene expression knockdown was carried out using the Dharmacon SMARTvector 2.0 system (Thermo Fisher Scientific, http://www.thermofisher.com) targeting IL8, according to the manufacturer's protocol. SMARTvector 2.0 Non-targeting Control Particles were used alongside the silencing constructs targeting IL8 for all downstream experiments.

\section{Protein Extraction and Western Blotting \\ Detailed in Supporting Information.}

\section{Statistical Analysis}

All data are expressed as mean \pm SEM. Statistical significance of differences between groups was determined by using one-way ANOVA with the Student-Newman-Keuls post-test with Prism5 Graphpad software.

\section{RESUlTS}

\section{OECs Have a Finite Replicative Lifespan In Vitro}

Human OECs were isolated following well-established protocols $[5,11]$. OECs were characterized as highly proliferative cells that grow as a monolayer with cobblestone-like morphology (Fig. 1A), form adherens junctions through cadherin proteins (Fig. 1B), and express endothelial markers VEGFR2 (Fig. 1C) and caveolin 1 (Fig. 1D). Furthermore, immunophenotypic analysis by flow cytometry (Fig. 1E) indicates this EPC subpopulation does not contain any hematopoietic cells (negative for CD45 and CD14), it is mainly composed of cells of endothelial lineage ( $\geq 99 \%$ positivity for CD31, CD105, and CD146), and also expresses the progenitor cell marker 

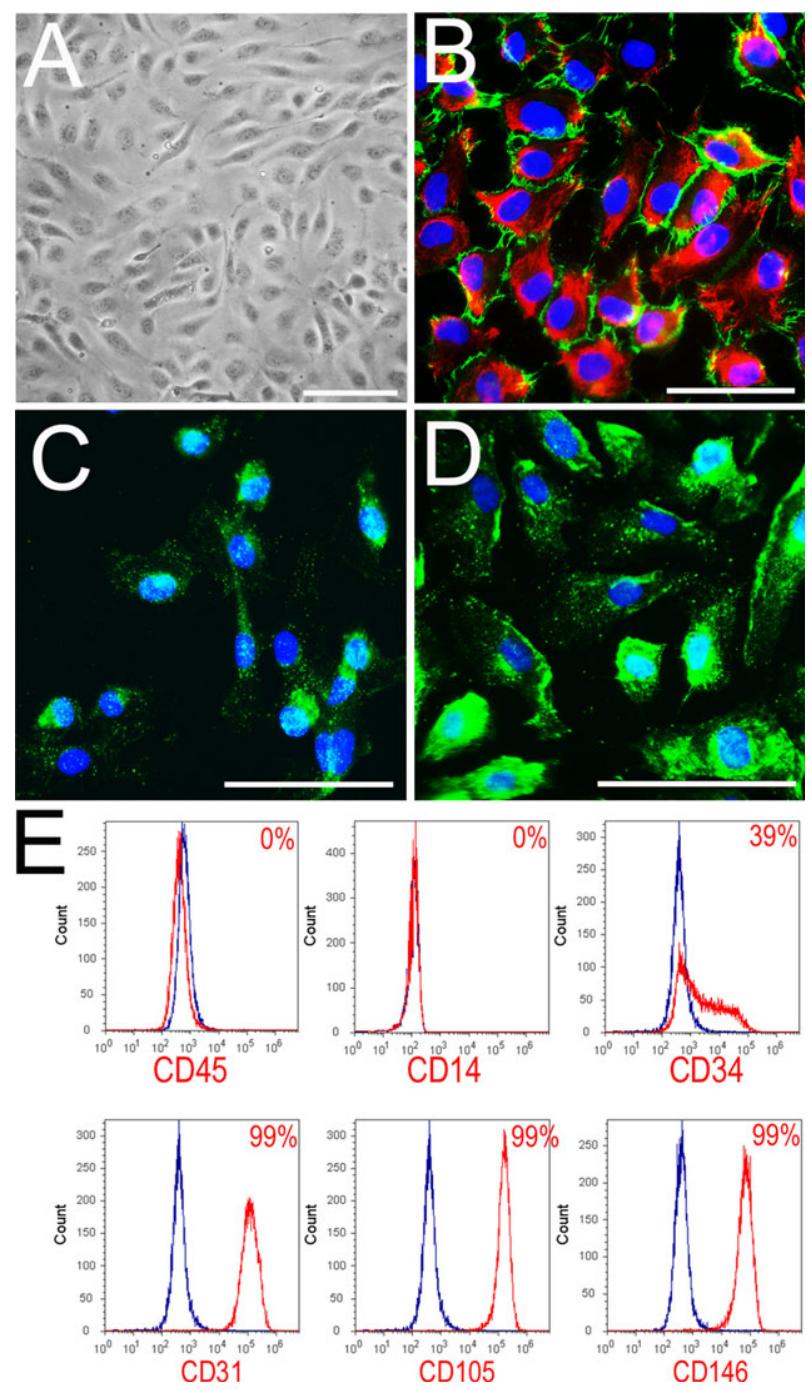

Figure 1. Characterization of endothelial progenitor cells as the outgrowth endothelial cell (OEC) population. (A): OECs grow in culture as a cobblestone-shaped cell monolayer. Scale bar $=100 \mu \mathrm{m}$. (B): Immunofluorescence staining for Vimentin (red) and cadherin (green). Nuclei are counterstained with 4',6-diamidino-2-phenylindole (DAPI) (blue). (C): VEGFR2 expression is shown in green. (D): Caveolin 1 expression in OECs is shown in green. Scale bars in (B-D) = $50 \mu \mathrm{m}$. (E): Immunophenotypic characterization of OECs by flow cytometry. Cell surface antibodies are shown in red histograms, respective isotype controls in blue histograms. The percentage of positive cells appears in the top right of each panel.

CD34 (albeit with variation according to passage number). Human OECs have been previously characterized at the transcriptome, flow cytometric, and ultrastructure levels [10, 11]. To evaluate the growth kinetics and replicative potential of OECs, growth curves were generated following strict recording of cell numbers during serial passage of adherent cultures (Fig. 2A). As expected for progenitors, all OEC clones showed considerably enhanced replicative potential when compared to human aortic endothelial cells and human dermal microvascular endothelial cells (HDMECs), which were used as differentiated mature counterparts. CB-OECs exhibited greater proliferative capacity than $\mathrm{PB}-\mathrm{OEC}$ with the former demonstrating, on average, 60 population doublings in 70 days, compared with PB-OECs which reached their Hayflick limit at 27 population doublings in 40 days. By contrast, mature endothelial cells displayed only 10 population doublings in 30 days.

Morphological assessment indicated a significant increase in cellular size during ex vivo expansion (Fig. 2B). Late-passage cells showed a fourfold enlargement of the cellular surface area (Supporting Information Fig. S1A) and an increase in cellular diameter occurred in unison with population doubling time (Fig. 2C). This physical enlargement of late-passage cells was even evident after monolayer trypsinization, with a significant increase in volume observed at the single cell level $(p<.001)$ (Fig. 2D).

The percentage of cells retaining the ability to synthesize DNA and incorporate BrdU (Fig. 2E) decreased significantly from $13 \%$ to $<2 \%(p<.001)$ (Fig. $2 \mathrm{~F})$. In addition, DNA content analysis using flow cytometry indicated that most late-passage OECs were arrested in the G1 phase of the cell cycle (Fig. 2G). When compared to early-passage OECs, late-passage OECs showed a significant decrease in the percentage of cells at $\mathrm{S}$ and $\mathrm{G} 2 / \mathrm{M}(p<.001)$ (Fig. $2 \mathrm{H})$. Collectively, these data demonstrate that although OECs can be efficiently expanded in vitro, their replicative potential is limited. After long-term expansion of OECs, cell numbers reach a plateau exemplified by the Hayflick limit where most cells are growth-arrested in G1 and have acquired an enlarged flattened morphology.

\section{OEC Growth Arrest Induced by Continuous Culture Ex Vivo Is Replicative Senescence}

Since cellular growth arrest can be defined as quiescence or senescence, our next aim was to identify which process was contributing to OEC growth arrest ex vivo. Contrary to quiescence, senescence is an irreversible state and cells typically show a complete loss of proliferative potential. Despite appropriate growth stimuli, such as availability of nutrients and space, OEC proliferation eventually became completely halted (Fig. 1A), which was suggestive of a senescence process. To confirm this, we measured SA- $\beta$-Gal activity at $\mathrm{pH} 6$, which is currently the most commonly used biomarker for senescence. Late-passage OECs showed a marked increase in SA- $\beta$-Gal activity when compared to early-passage counterparts (Fig. $3 \mathrm{~A})$. Quantification indicated a significant increase in the percentage of cells that were positive for SA- $\beta$-Gal; $3 \%$ in earlypassages increasing to $60 \%$ in late-passages (Fig. 3B). SA- $\beta$ Gal activity is associated with expansion of the lysosomal mass [12]; therefore a lysotracker probe was used. Consistent with SA- $\beta$-Gal data, there was a significant fourfold increase in the lysosomal mass per cell when comparing early- to latepassage OECs (Supporting Information Fig. S1B, S1C). DNA damage is a cardinal feature of cellular senescence, and the presence of the established DNA damage marker $\gamma$-H2AX was evaluated during the replicative lifespan of OECs (Fig. 3C). A significantly higher percentage of OECs positive for $\gamma$-H2AX was observed in the late-passage group when compared to early-passage cells $(p<.001)$ (Fig. 3D).

Senescence is associated with major changes in gene expression therefore we performed transcriptome analysis. Total RNA was extracted from early- and late-passages for the same OEC clone, and three biological replicates were used for transcriptome analysis using the Illumina WG-6 v3.0 expression beadchips to assay more than 48,000 transcripts. Statistical assessment using National Institute on Aging array analysis distinguished a significant differential gene expression when comparing early- and late-passages (Fig. 3E). Overall, 782 transcripts were found to be significantly upregulated in early-passage OECs, while 654 transcripts were significantly upregulated in late-passage OECs. Differences in gene signatures were consistent among three different 

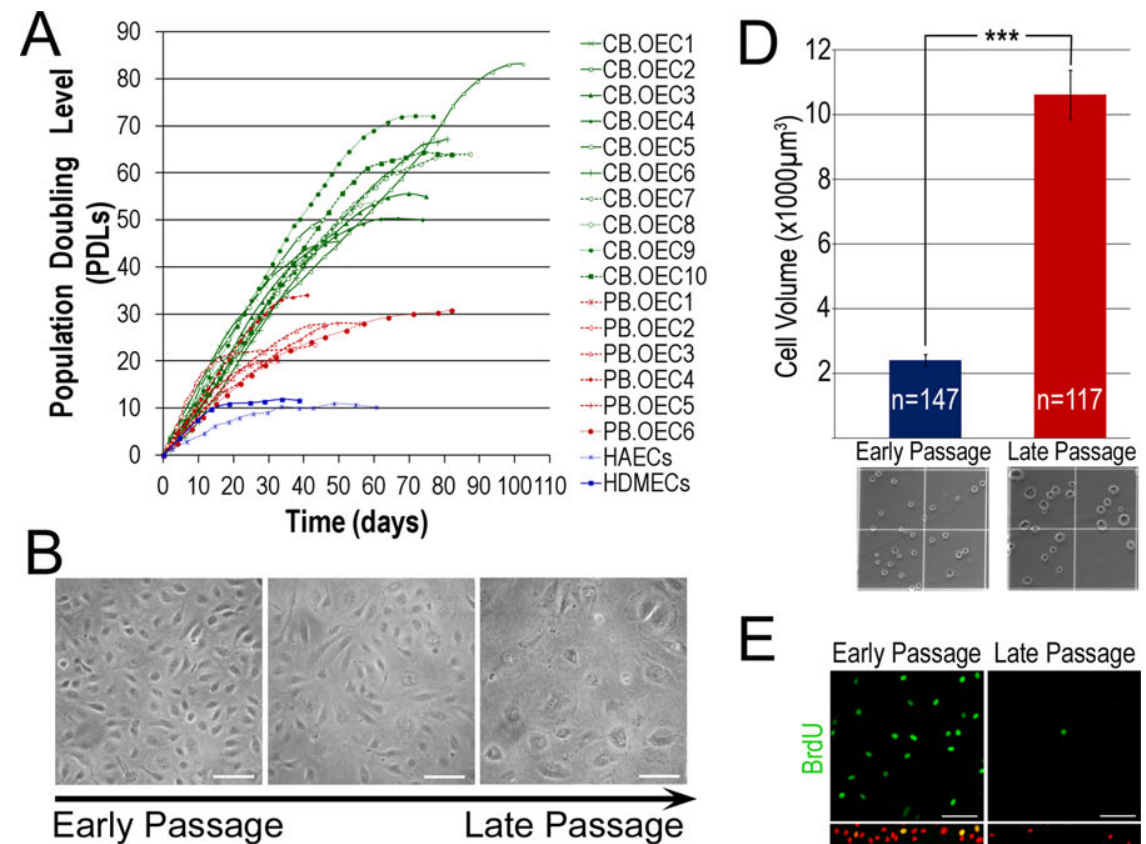

E

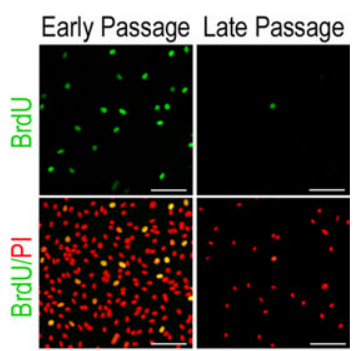

C

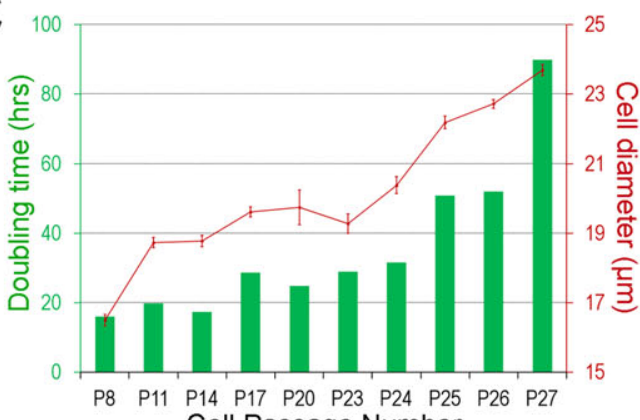

G

Cell Passage Number
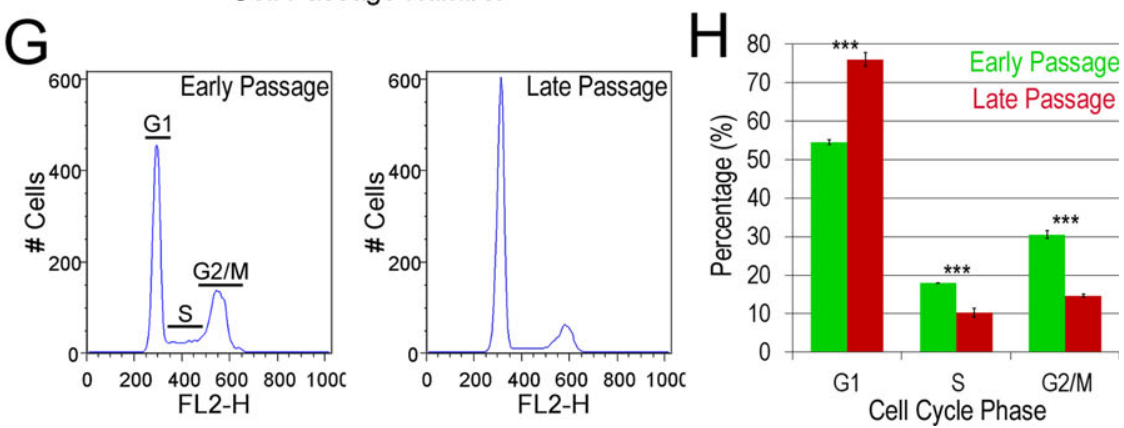

Figure 2. OECs exhibit a finite replicative lifespan in vitro. (A): Growth curves for ex vivo expanded cord blood-derived OECs (green), peripheral blood-derived OECs (red), human aortic endothelial cells (HAECs) and human dermal microvascular endothelial cells (HDMECs) in blue. (B): Representative phase-contrast micrographs of cultured OECs at different stages during their in vitro lifespan. Scale bars $=100 \mu \mathrm{m}$. (C): Concomitant increase in doubling time and cell diameter with cellular passage. (D): Comparison of cell volume between early-passage and late-passage OECs. Bottom panel shows representative phase-contrast micrographs of OECs in the hemocytometer chamber. (E): Immunofluorescence staining for BrdU shown in green, nuclei are counterstained in red with PI. Scale bars $=100 \mu \mathrm{m}$. (F): Quantification and statistical analysis comparing BrdU incorporation in early- and late-passage OECs $(n=4)$. $(\mathbf{G})$ : Cell cycle analysis using flow cytometry and PI demonstrates growth arrest at the G1 phase of the cell cycle in late-passage OECs. (H): Quantification of cell cycle analysis comparing early and late-passage OECs. Late-passage OECs had significantly fewer cells in the S and G2/M phase, while exhibiting a significant increase in the G1 phase $(n=3)$. The error bars indicate standard error; ***, $p<.001$. Abbreviations: BrdU, bromodeoxyuridine; $\mathrm{CB}$, cord blood; HAEC, human aortic endothelial cell; HDMEC, human dermal microvascular endothelial cells; OEC, outgrowth endothelial cell; PB, peripheral blood; PI, propidium iodide.

biological replicates, as shown in heatmaps for the most altered genes (Fig. 3F). Quantitative reverse transcriptase polymerase chain reaction (qRT-PCR) was used to validate microarray results and showed comparable changes (Fig. 3G). In order to gain an insight into the biological meaning behind this long list of genes, gene ontology analysis for biological processes was performed using the functional genomics tool FunNet. This highlighted that while gene ontologies for transcripts significantly upregulated in early-passage OECs were associated with cell cycle, mitosis, and DNA replication; gene 


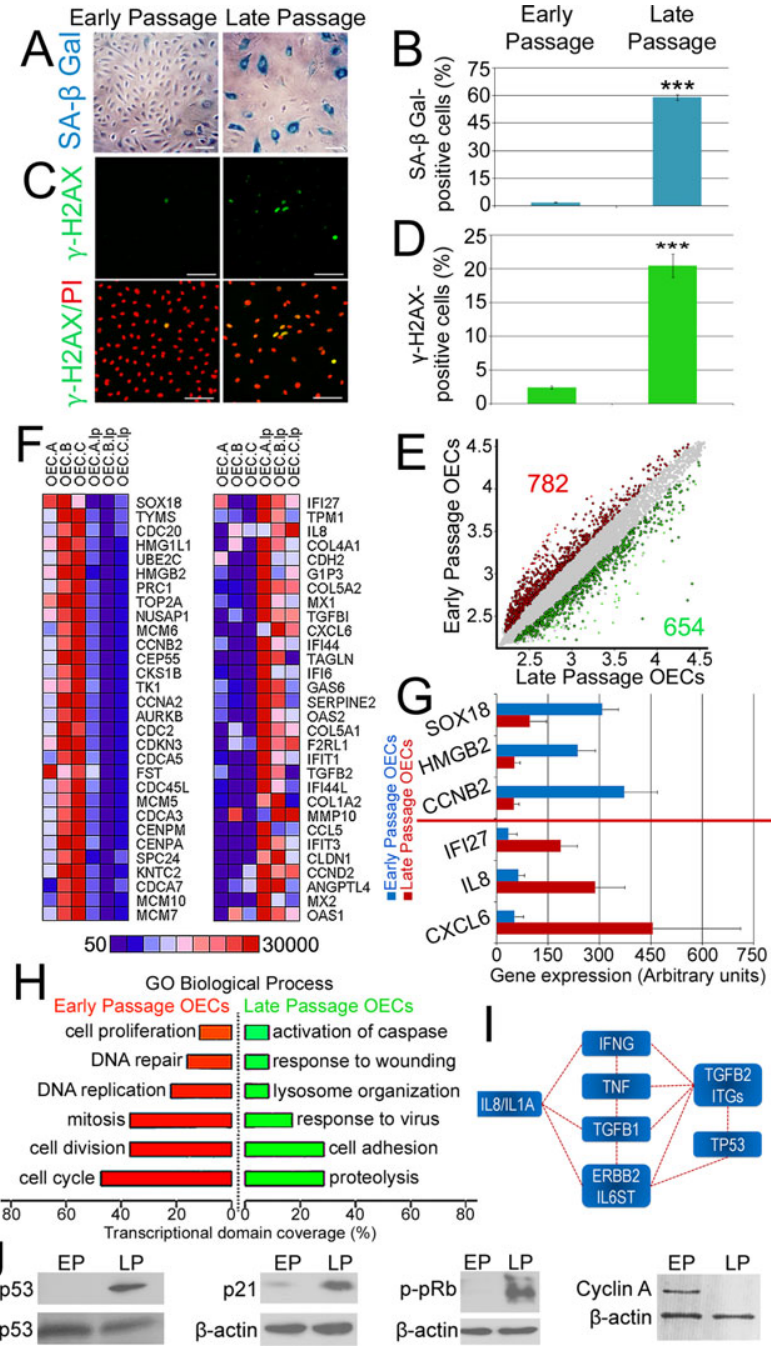

Figure 3. OEC growth arrest induced by ex vivo expansion is a result of cellular senescence. (A): Representative images for earlyand late-passage OECs stained for SA- $\beta$-Gal shown in blue. Scale bars $=100 \mu \mathrm{m}$. (B): Statistical analysis comparing percentages of SA- $\beta$-Gal positive cells in early-passage versus late-passage OECs ( $n$ $=4)$. (C): Representative immunofluorescence staining for DNA damage marker $\gamma$-H2AX in green. Nuclei are counterstained in red with PI. Scale bars $=100 \mu \mathrm{m}$. (D): Statistical analysis comparing percentages of $\gamma$-H2AX positive cells in early-passage versus late-passage OECs $(n=4)$. (E): Dot plot for genome-wide transcriptome analysis using National Institute on Aging array with false discovery rate $(\mathrm{FDR})=0.05$ to compare early-passage with late-passage OECs. 782 transcripts were significantly upregulated in early-passage OECs (shown as red dots), while 654 transcripts were significantly upregulated in late-passage OECs (green dots). (F): Heat map for the top 30 upregulated and downregulated transcripts demonstrates a consistent gene expression change among paired biological replicates $(n=3)$. (G): Real time quantitative reverse transcriptase polymerase chain reaction for SOX18, HMGB2, CCNB2, IFI27, IL8, and CXCL6 serve to show consistent alterations between early and late-passage OECs and validate gene microarray results. (H): GO assessment using the functional genomics tool FunNET. (I): Molecular pathway network analysis using Ingenuity software for assessment of transcripts upregulated in late-passage OECs. (J): Western Blotting to evaluate expression of phospho-p53, p21, pRb, and cyclin A in early- and late-passage OECs. The error bars indicate SE; ***, $p<.001$. Abbreviations: EP, early passage; GO, gene ontology; IL, interleukin; LP, late passage; OEC, outgrowth endothelial cell; PI, propidium Iodide; SA- $\beta$-Gal, senescence-associated $\beta$ galactosidase activity. ontologies associated with late-passage OECs were proteolysis, cell adhesion, and lysosome organization (Fig. 3H). Similar results were obtained when data were processed using visANT software (Supporting Information Fig. S2). Further analysis using Ingenuity software identified molecular pathways associated with OEC senescence which included p53, transforming growth factor (TGF), tumor necrosis factor (TNF), interferon (IFN), and inflammatory cytokines IL8, IL1A, and IL6 (Fig. 3I). To examine the possibility of endothelial differentiation during in vitro expansion of progenitors, the transcriptome was assessed for variations in gene expression of endothelial differentiation and progenitor markers. Interestingly, we found that while progenitor markers expression such as SOX18 and CD34 decreased in late passages, endothelial differentiation markers such as CDH5 and ESM1 increased in late passages (Supporting Information Fig. S3A). Consistent with these findings, flow cytometry analysis revealed that CD34 expression significantly decreased from $72 \%$ in early passages to $13 \%$ in late passages. Similarly, ckit expression significantly decreased from $23 \%$ to $2 \%$ between early and late passages, respectively (Supporting Information Fig. S3B). Together these findings, at the transcriptome and protein expression level, suggest that senescence triggered by in vitro expansion is coupled to endothelial differentiation. Nevertheless, results from long-term cell number amplification in vitro indicate that the high proliferative potential of OECs is a useful feature that allows them to be clearly distinguished from mature endothelial cells (Fig. 2A).

Classic cellular senescence is driven by tumor suppressor pathways p53 and pRb, therefore expression of these proteins was investigated (Fig. 3J). While there was no difference in the expression of total p53, a clear increase in phosphorylated p53 was observed in late-passage OECs. In addition, p21 and $\mathrm{pRb}$ were consistently upregulated in late-passage OECs, while cyclin A was only found in early-passage OECs.

Having established that late-passage OECs are senescent, we investigated if the $\mathrm{OEC}$ senescence program during ex vivo expansion was associated with telomere erosion as a result of continuous cell division in culture. Flow cytometry and fluorescence in situ hybridization (FISH) were used to measure the average length of telomeres from the same clones at different passages. Dot plots illustrated fluorescence measurements of cells incubated with FITC-conjugated telomere-specific peptide nucleic acid (PNA) probes were compared to cells incubated with the negative controls. Overlap of populations was found only in senescent cells (Fig. 4A) indicating that these OECs had shorter telomeres. Further quantification by evaluating mean fluorescence intensities in histograms (Fig. 4B) highlighted significant telomere shortening in senescent OECs when compared to early-passage OECs $(p<.001)$ (Fig. 4C). Telomerase, shown to be highly expressed in cancer and stem cells, extend telomeres after each round of cell division. We used the telomere repeat amplification protocol (TRAP) assay to detect telomerase activity in early-passage and senescent OECs. The myeloid cell line U937 was used as a control for high telomerase activity while the primary isolates of HDMECs served as control for low telomerase activity. In agreement with previous results, decreased telomerase activity was observed in senescent OECs from all the clones (Fig. 4D) thereby providing further evidence that OECs undergo replicative senescence during expansion.

\section{Cellular Senescence Limits the Regenerative Potential of OECs}

While there is evidence linking cellular senescence to both stem cell and endothelial cell dysfunction, there is remarkably little known about how senescence affects OEC functionality. 

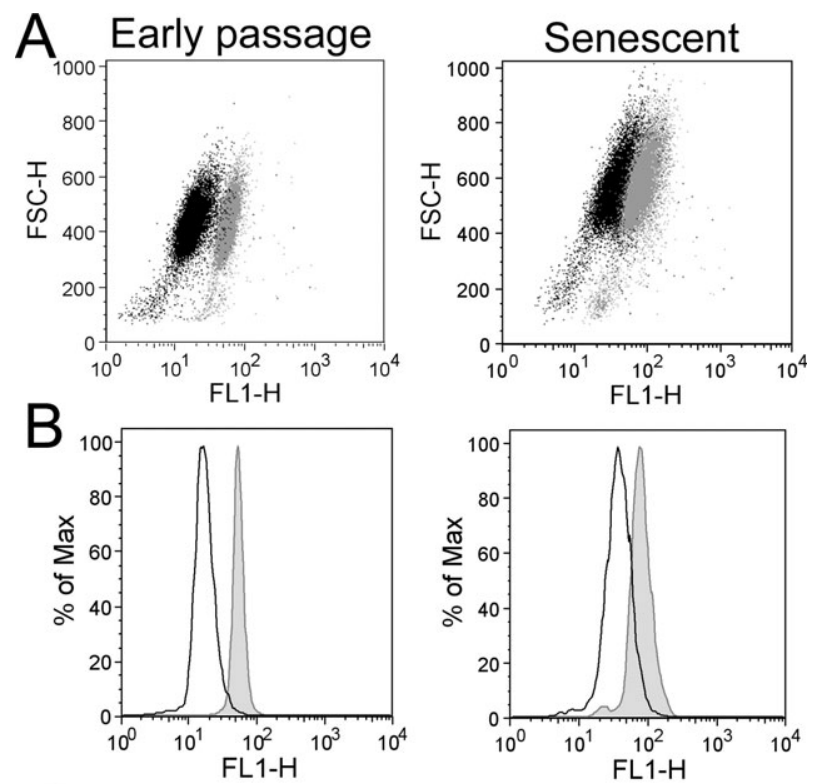

C

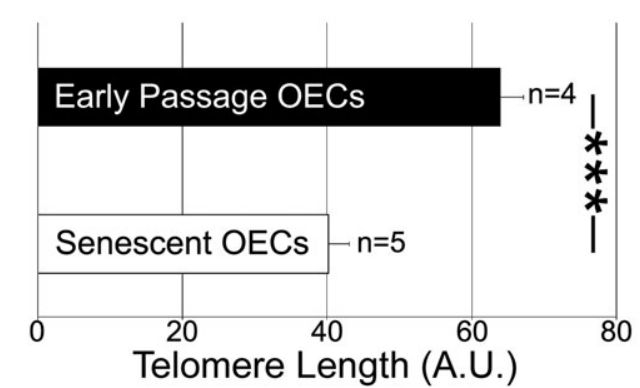

D

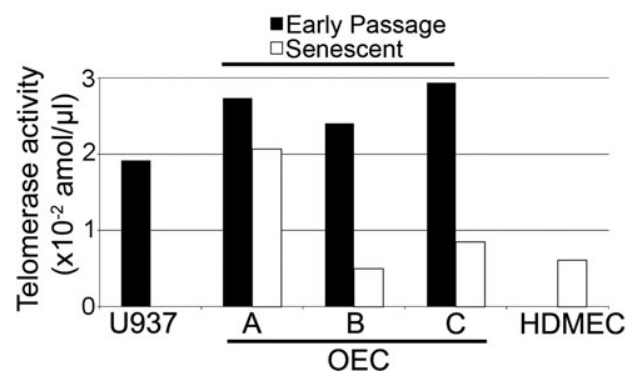

Figure 4. Telomere shortening as a hallmark of replicative senescence in OECs. (A): Telomere length was determined by flow cytometry using fluorescence in situ hybridization and a fluorescein-conjugated peptide nucleic acid probe. Representative dot plots show two distinct cell populations which have been incubated with and without an fluorescein isothiocyanate (FITC)-conjugated peptide nucleic acid probe specific for telomere repeats. These are shown as gray and black dots, respectively. Note that overlap only exists in senescent cells. (B): Representative fluorescence intensity histograms show differences in mean fluorescence intensities between telomere probes and control probes (gray filled histograms and white histograms, respectively). Note the larger distance between peaks in the early-passage OEC group compared to the late-passage group. (C): Quantification of telomere length by normalizing distances between mean fluorescent intensities to arbitrary units (A.U.). Statistical analysis to compare early-passage and senescent OECs. (D): Telomerase activity was assessed using the telomere repeat amplification protocol (TRAP) assay for pairs of OEC clones $(n=3)$. Note that there is a significant decrease in telomerase activity when comparing senescent OECs to their early-passage counterparts. The U937 cell line was used as a positive control for high telomerase activity, and HDMEC provided an appropriate control for low telomerase activity. The error bars indicate SEM. $* * *, p<.001$. Abbreviations: HDMEC, human dermal microvascular endothelial cells; OEC, outgrowth endothelial cell.
Thus, we examined whether replicative senescence impairs functional properties of OECs. First, we used a scratch wound assay to compare migratory capacities of early-passage and senescent OECs. Eight hours after creating the wound, earlypassage OECs almost closed the gap while senescent OECs show much reduced capacity (Fig. 5A). Quantification of migrated areas corroborated this qualitative data $(p<.001)$ (Fig. 5B). Second, the Oris cell migration assay was used. In agreement with the scratch wound assay, we found impairment in the migratory capacity of senescent OECs (Fig. 5C), which was significantly lower than that of early-passage OECs $(p<.001)$ (Fig. 5D). To test tubulogenic potential, a Matrigel-based 3D angiogenesis model was performed (Fig. 5E). After 72 hours, it was evident that in vitro tube forming capacity was significantly impaired in senescent OECs $(p<.001)$ (Fig. 5F).

The impact of OEC senescence on their vasoreparative (therapeutic) potential was investigated in vivo using the OIR model. OIR provides a consistent and reliable preclinical system to test the therapeutic benefit of a cytotherapy by objectively assessing vascular repair in the ischemic retina. Mouse pups consistently developed vaso-obliteration of the central retina at P12 (Fig. 5G). At P13, mice were divided into three experimental groups ( $n=6$ animals per group) for the delivery of equal numbers of early-passage OECs, senescent OECs, or vehicle. Cells were prelabeled with fluorescent Qdots and delivered via intravitreal injection. Retinas were sampled at 72 hours and vascular areas were evaluated by Isolectin staining (Fig. 5G). Injection of both early- and latepassage OECs resulted in a significant reduction of the avascular area $(p<.001)$. Although senescent OECs were still beneficial, they were less vasoreparative than their normal counterparts (Fig. 5H). Despite the observed reduction in retinal ischemia, it was apparent that most senescent OECs did not integrate into the resident vasculature as efficiently as the early-passage OECs. Further quantification of preretinal pathological neovascularization showed that only the earlypassage OECs significantly decreased the amount of neovascular tufts $(p<.001)$ (Fig. 5I). Quantification of neovascular areas confirmed that there was no difference among the groups treated with senescent OECs and vehicle (Fig. 5I). These findings indicate that replicative senescence not only affects the proliferative potential of OECs but also their migratory and tubulogenic capacities. Most importantly, when used as a cytotherapy for vascular repair, senescent OECs do not perform as well as their presenescent counterparts.

\section{Senescence Program of OECs Has a Major Inflam- matory Component}

Key factors involved in OEC senescence were identified using transcriptomic analysis. Ingenuity Pathway software determined "Inflammatory Response" as the top BioFunction in senescent OECs. Alongside this, the resultant gene network established for transcripts upregulated in senescence included many inflammatory cytokines, such as IL8, IL1A, IL32, IL6ST, and CXCL1 (Supporting Information Fig. S4A). This was confirmed using REVIGO software which highlighted "Regulation of cytokine production" in the center of the semantic space for senescent OEC transcripts (Supporting Information Fig. S4B), suggesting an important role for inflammatory cytokines in modulation of senescence-upregulated genes. Ingenuity software also recognized STAT3, STAT2, and TP53 as transcription factors associated to senescent OECs upregulated genes. This was confirmed by Genomatix software, which assessed promoters of senescence-upregulated genes, indicating STAT1, STAT2, STAT3, TP53, and NF $\kappa \mathrm{B}$ 
as relevant transcription factors. All this in silico analysis of transcriptomic data, using a diverse range of bioinformatics software, indicated a strong link between OEC replicative senescence and inflammation-related pathways. Therefore, we next compared gene expression of inflammatory cytokines by qRT-PCR in early-passage versus senescent OECs. Although no difference was found in gene expression of the $\mathrm{NF} \kappa \mathrm{B}$ components rela and $i k k b$, there was a significant upregulation of IL8,ILIB, and ILIA (Supporting Information Fig. S5). To confirm these results at the protein level, an angiogenesis proteome profiler was used. Proteins with increased expression in senescent OECs included IL8 and IL1B (Fig. 6A).

Recently, the senescence-associated secretory phenotype (SASP) [13] or senescence messaging secretome [14] has been included as a hallmark of cellular senescence. This information prompted the characterization of the secretome in senescent OECs. A cytokine proteome profiler was used to identify and quantify proteins present in the conditioned media of early-passage and senescent OECs. The most abundant protein in the senescent OEC secretome was IL8, which was expressed to a similar level as positive controls (Fig. 6B). Densitometric analysis confirmed a significant fivefold increase in IL8 when con-

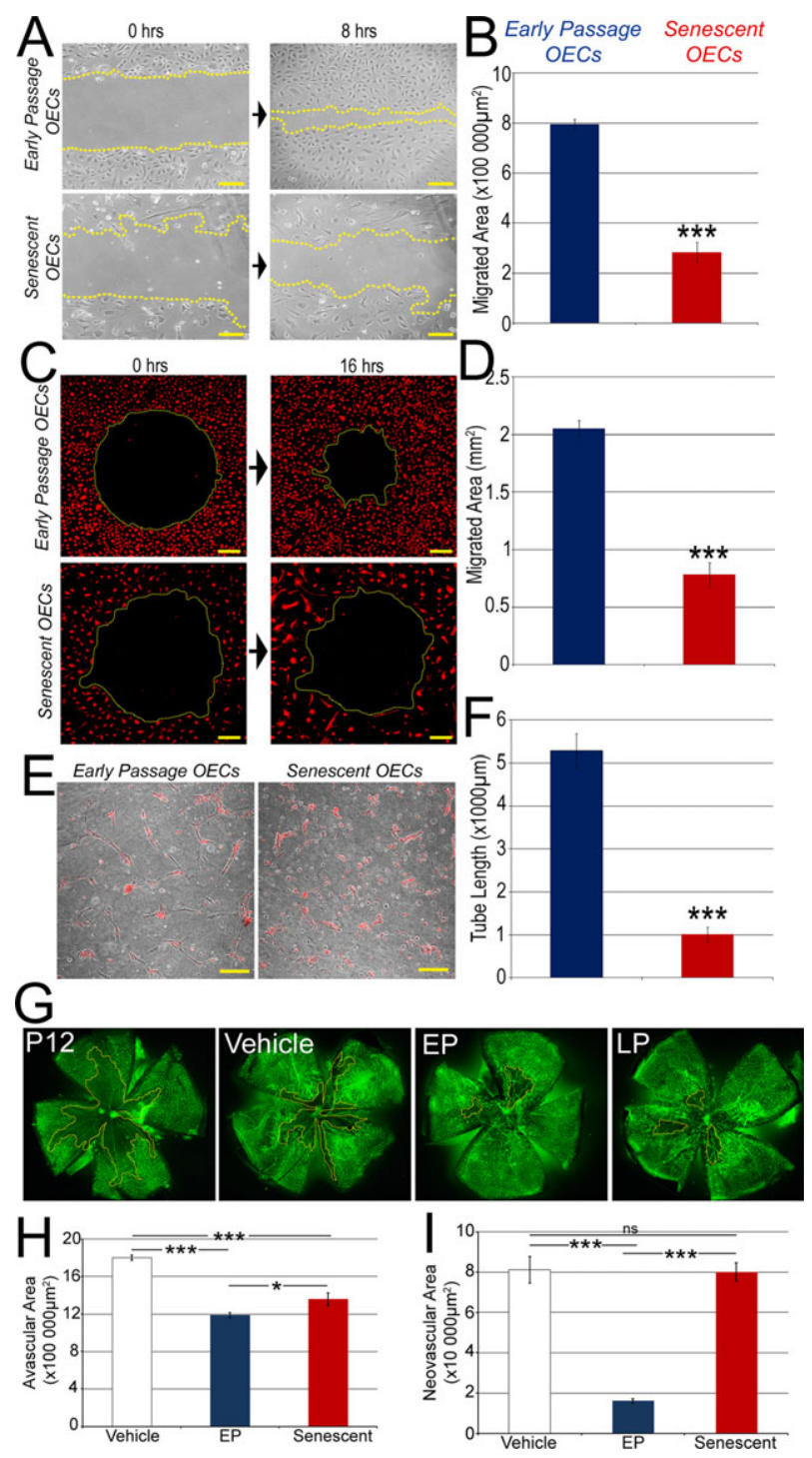

Figure 5. ditioned media from senescent OECs was compared to conditioned media of early-passage OECs $(p<.001)$ (Fig. 6C). Other proteins shown to increase in the secretome during senescence were CXCL1, sICAM-1, and IL6; proteins that were present at similar levels were MIF and CCL2; while PAI-1 expression decreased in senescent secretomes (Supporting Information Fig. S6). Quantitative ELISA corroborated a significant increase in the IL8 levels secreted per cell when comparing early-passage to senescent OECs $(p<.001)$ (Fig. 6D). Limulus amebocyte lysate (LAL) endotoxin testing confirmed that cell culture conditions at both early and late-passages were free of bacterial endotoxins or lipopolysaccharide (LPS) (Supporting Information Fig. S7).

These results at transcriptome, proteome, and secretome levels indicate that there is a clear association between inflammatory cytokines and OEC replicative senescence. This suggests a possible role for some of these cytokines in the induction, establishment, or reinforcement of OEC senescence.

\section{IL8 Depletion Delays Replicative Senescence in Cultured OECs}

A network of inflammatory cytokines was found to be significantly upregulated in senescent OECs and it was therefore important to establish if these might play a role in the senescence program of these cells. Our investigations focused on IL8 since it was consistently among the most differentially expressed mRNA and the most highly expressed cytokine in the secretome of senescent OECs. The SMARTvector 2.0 lentiviral shRNA technology was used to knockdown IL8 expression in OECs with approximately $93 \%$ transduction efficiency after Puromycin selection (Supporting Information Fig. S8A, S8B). qRT-PCR confirmed a significant decrease in IL8 mRNA when shRNA-IL8 transduced cells were compared to

Figure 5. Replicative cellular senescence limits OEC regenerative potential by impairing vasoreparative properties. (A): Representative phase-contrast micrographs for the scratch wound assay on early-passage and senescent OECs. Wound edge delineated with yellow dots. (B): Quantification of migrated areas using NIS elements software ( $n$ $=10)$. Note significant impaired migration in senescent OECs. (C): Oris Cell Migration Assay with OECs prestained using the vital dye calcein red. The central area where cells migrated is outlined in yellow. (D): Quantitative analysis of migrated areas $(n=12)$. Note significantly smaller migrated areas in the senescent OEC group. (E): The tube-forming capacity of OECs was assessed using the 3D-angiogenesis model. Representative images of OECs embedded in Matrigel 7 days after starting the assay. Calcein staining in red. (F): Quantification of tube length per field of vision $(n=4)$ shows significantly decreased tube forming capacity in senescent OECs. (G): The oxygen induced retinopathy model was used to determine the therapeutic potential of OEC cytotherapy. Murine retinal flat mounts were stained using isolectin (green) to identify retinal vasculature and multiple images per retina with magnification $\times 10$ were taken and stitched together using NIS elements software. The central avascular area is shown in black and delineated with a yellow line. Images at postnatal day 12 (P12) shows the ischemic state of retinas at the point of OEC delivery by intravitreal injection. All other images were taken 72 hours after cell or vehicle delivery. (H): Quantification of the avascular area of retinas 72 hours after intravitreal injection with either vehicle, EP-OECs, or LP-OECs $(n=6)$. Note senescent OECs significantly decrease avascular areas but with less efficiency than early-passage counterparts. (I): Quantification of the pathological preretinal neovascularization 72 hours after intravitreal injection of OECs $(n=6)$. In contrast to early-passage OECs, note that senescent cells fail to significantly decrease pathological neovascularization. The error bars indicate SEM. *, $p<.05$; ***, $p<.001$; ns, not significant. Scale bars $(A, C, E)=250 \mu \mathrm{m}$. Abbreviations: EP, early passage; LP, late passage; OEC, outgrowth endothelial cell. 

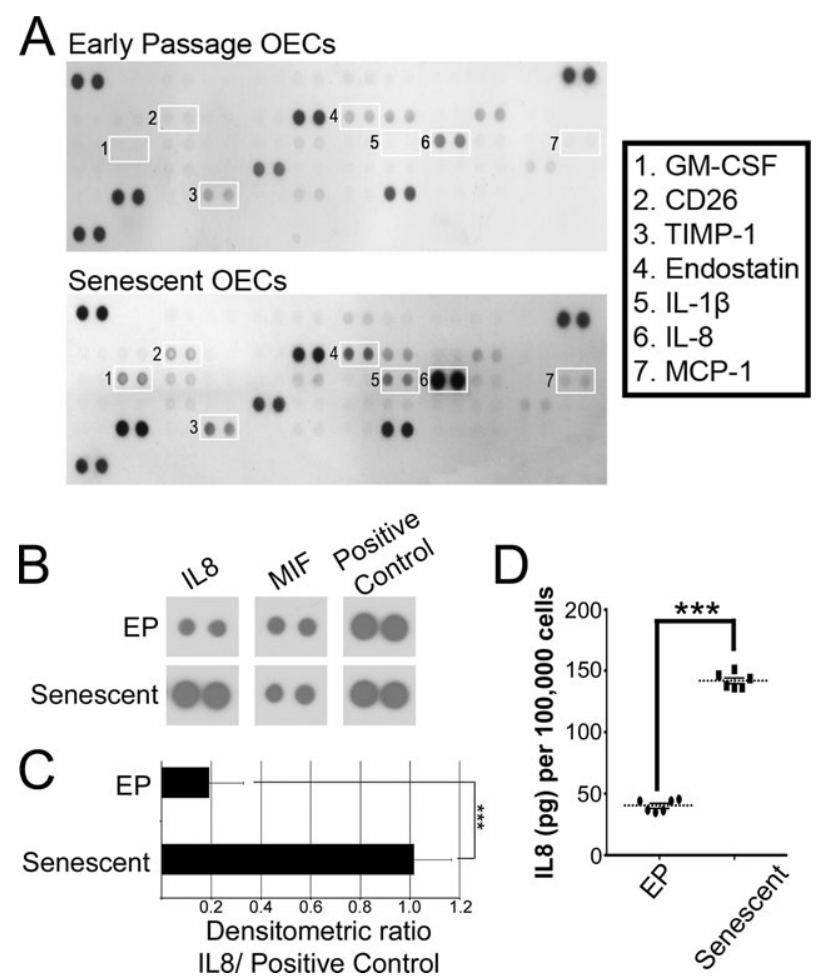

Figure 6. Inflammatory component identified in OEC senescence program. (A): Protein lysates from early-passage and senescent OECs were assessed using the human angiogenesis proteome profiler. Senescent OECs showed increased expression of many proteins (highlighted by black box). Positive controls are included in the top corners and bottom left corner and negative controls in the bottom right corner. (B): Human cytokine proteome profiler for evaluation of conditioned media from early-passage and senescent OECs. Note higher IL8 secretion from senescent cells while no changes are observed in MIF and positive controls. (C): Quantification of cytokine array by densitometry using ImageJ software $(n=4)$. (D): ELISA quantification of IL8 levels in conditioned media from early-passage and senescent OECs $(n=6)$ shows higher levels in senescent cells. The error bars indicate SEM. $* * *, p<.001$. Abbreviations: EP, early passage; GM-CSF, granulocyte macrophage colony stimulating factor; IL, interleukin; OEC, outgrowth endothelial cell; MCP, monocyte chemotactic protein; MIF, macrophage migration inhibitory factor; TIMP, tissue inhibitor of metalloproteinase.

shRNA-controls (Supporting Information Fig. S8C). We next explored the presence of cytokines in the secretome of OECs transduced with shRNA-IL8 and shRNA-control. As expected, there was a significant decrease of IL8 expression in the shRNA-IL8 group to $16 \%$ of the IL8 levels found in the shRNA-control group (Fig. 7A). Interestingly, we found that depletion of IL8 concomitantly lead to a significant decrease in the expression of MCP-1 and IL6, while there were no changes in the expression of PAI-1, MIF, and CXCL1 (Supporting Information Fig. S9). Further quantification of conditioned media by ELISA indicated that shRNA-control and nontransduced OECs have similar IL8 levels, while there was a significant decrease from $1,208 \mathrm{pg} / \mathrm{mL}$ in shRNA-control OECs to $101 \mathrm{pg} / \mathrm{mL}$ in shRNA-IL8-transfected OECs $(p<$ .001) (Fig. 7B).

In order to determine the potential regulatory role of IL8 in the OEC senescence program, growth curves for shRNAIL8 and respective shRNA-control were generated. IL8 depletion markedly delayed OEC senescence during ex vivo expansion, as shown by more than twofold increase in accumulated population doublings and protracted exponential phase of the growth curve (Fig. 7C). Clonogenic assays corroborated results from growth curves, larger sized and greater numbers of single cell-derived colonies occurred in the shRNA-IL8 group $(p<.001)$ (Fig. 7D). Analysis of SA- $\beta$-Gal revealed a significant decrease in the number of OECs positive for this senescence biomarker when IL8 was depleted $(p<.001)$ (Fig. 7E). This is consistent with a marked decrease in DNA damage in IL8-depleted OECs as shown by immunocytochemistry for 53BP1 nuclear foci (Supporting Information Fig. S10).

To assess the impact of IL8 on senescence-related loss of functionality, Matrigel-based tubulogenesis assays were used. shRNA-mediated depletion of IL8 restored tube forming potential as indicated by the significant increase in the number of branching points when shRNA-IL8 OECs were compared to shRNA-control OECs at the same passage ( $p$ $<$.001) (Fig. 7F). In agreement with this, the migratory capacity of late-passage OECs was significantly enhanced $(p<.001)$ when IL8 was depleted (Fig. 7G). These results provide evidence of a role for IL8 during OEC replicative senescence since attenuating expression of this cytokine not only delayed senescence but also increased proliferation rate, extended in vitro lifespan, and enhanced functionality.

We also investigated the functional role of shRNA-IL8 OECs in an in vivo model of ischemic retinopathy. Interestingly, these transplanted, virally modified OECs induced a marked inflammatory cell infiltration response when compared to vehicle-injected controls in the contra-lateral retina (Supporting Information Fig. S11A). Myeloid cells accumulated preferentially around endothelial tubes formed by shRNA-IL8 OECs (Supporting Information Fig. S11B). This finding underscores the importance of establishing the immunogenic potential of genetically modified OECs before their usage in cell therapies and is currently being investigated by our group.

As IL8 has been previously described as a mediator of angiogenesis, its effects on the OEC angiogenic program were dissected and studied at three distinct cellular passages prior to the onset of senescence. OEC treatment with IL8 varied from 1 to $100 \mathrm{ng} / \mathrm{mL}$ and proliferative potential decreased with cellular passage (Supporting Information Fig. S12A). However, IL8 did not increase colony-forming capacity in any group but significantly decreased proliferative potential when early-passage OECs were exposed to higher IL8 concentrations $(p<.05)$ (Supporting Information Fig. S12B). Results from OEC migration studies agreed with OEC colony formation data. Late passage OEC migration rate was lower than early passage counterparts (Supporting Information Fig. S12C), and there was only a significant decrease in migration areas when early-passage OECs were exposed to high IL8 doses $(p<.01)$ (Supporting Information Fig. S12D). In other passages, there was no significant effect when OECs were exposed to IL8. Assessment of OEC tube formation consistently indicated a clear decrease in tube forming capacity with cellular passage (Supporting Information Fig. S12E). Interestingly, while late-passage OECs do not respond to IL8 (Supporting Information Fig. S12F), we found a significant increase in tube length when earlyand mid-passage OECs were exposed to lower doses of IL8 $(p<.001)$. Exposure of OECs to exogenous recombinant human IL8 for 5 days did not affect their intrinsic senescence program (Supporting Information Fig. S12G, S12H). These findings indicate that a proangiogenic effect for IL8 is only found in early and mid-passage OECs and with low IL8 concentrations (1 $\mathrm{ng} / \mathrm{ml})$ as demonstrated by the tube formation assay. Late-passage OECs are clearly nonresponsive to IL8 pro-angiogenic effects. 

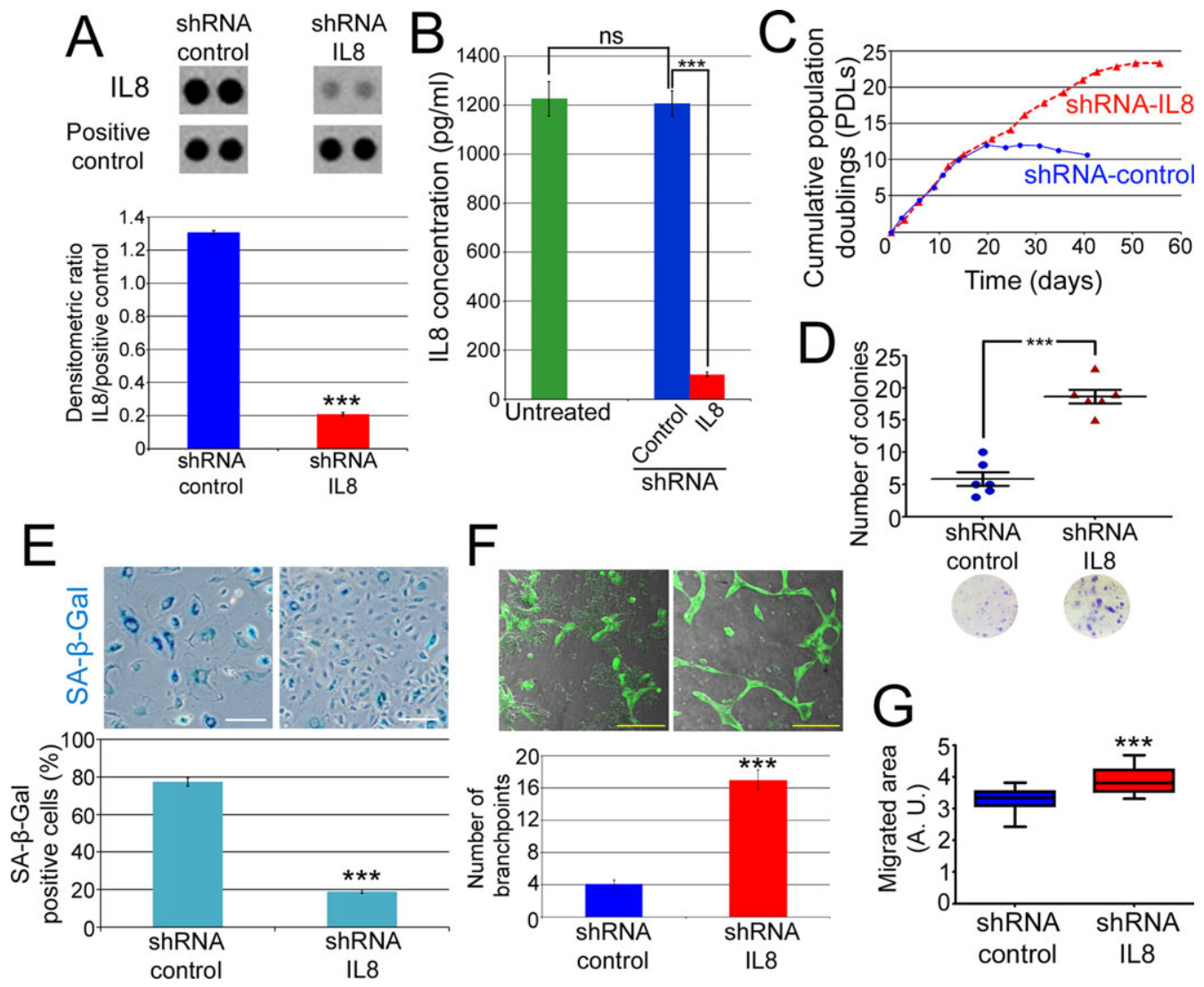

Figure 7. shRNA lentiviral transduction-mediated IL8 depletion delayed outgrowth endothelial cell (OEC) senescence. (A): The human cytokine proteome array was used to evaluate IL8 expression by assessing conditioned media of OECs transduced with either shRNA-control or shRNA-IL8 lentiviral constructs. Quantification of blots by densitometry $(n=4)$ shows efficient IL8 knockdown in OECs transduced with IL8shRNA. (B): ELISA quantification of IL8 levels in conditioned media of shRNA-control and shRNA-IL8 transduced OECs, compared to same passage nontransduced OECs $(n=3)$. (C): Growth curves for shRNA-control and shRNA-IL8 OECs shown in blue and red, respectively. (D): Clonogenic assay comparing colony formation potential of shRNA-control and shRNA-IL8 OECs $(n=6)$. Bottom panel shows representative image of cells stained with crystal violet to identify and count colonies. (E): SA- $\beta$-Gal assay 1 month after lentiviral transduction. Representative images showing higher expression (blue) in shRNA-control OECs. Scale bars $=100 \mu \mathrm{m}$. Quantification demonstrates significantly fewer cells positive for SA- $\beta$-Gal in the shRNA-IL8 group $(n=8)$. (F): Representative images of tube-like structures formed in the Matrigel 3D angiogenesis model. Cells stained in green with vital dye calcein. Quantification of number of branch points formed per field of vision $(n=12)$. Scale bars $=250 \mu \mathrm{m}$. (G): Quantitative analysis of migrated areas using the scratch wound assay shows impaired migration potential in shRNA-control OECs (blue) when compared to shRNA-IL8 OECs (red) $(n=12)$. The error bars indicate SEM. ***, $p<.001$; ns, not significant. Abbreviations: IL, interleukin; SA- $\beta$-gal, SA-senescence-associated $\beta$ galactosidase; shRNA, small hairpin RNA.

\section{DisCUSSION}

In this study, we provide evidence demonstrating the markedly enhanced replicative potential of OECs in comparison to mature endothelial cells. This characteristic favors the isolation of OECs and their possible use for cell therapy, since large numbers of cells are typically required and are essential for autologous/ allogeneic stratagems. A porcine model of acute myocardial infarction recently showed that efficient neovascularization of an individual ischemic heart required a minimum delivery of 30 million OECs [15], which is equivalent to approximately ten $75 \mathrm{~cm}^{2}$ confluent cell culture flasks. If we consider that a homogeneous OEC population is generally established in vitro after passage 3 with approximately 100,000 cells, then a minimum of eight population doublings is required to amplify cell numbers for therapeutic use. Our data shows that this is feasible for both $\mathrm{CB}$ - and PB-derived OECs, as shown by their respective growth curves. However, our observations also indicate that OECs expanded ex vivo ultimately undergo senescence upon exhaustion of their replicative potential. Critically, the current investigation has shown that the molecular fingerprint for senescence is coupled to a dysfunctional phenotype which underlines the importance of evaluating the senescence program when expanding progenitor cells for clinical use.

The evidence demonstrating that OECs undergo replicative senescence during expansion in culture underscores their safety when applied to human cytotherapy. Very recently, it has been noted that senescence in culture is the most reliable test for tumorigenicity [16], because assays using nude mice have unacceptably low sensitivity.

Although cytotherapy for vascular repair during ischemia using patients' own blood/bone marrow has been shown to be attainable [17], this autologous strategy faces significant limitations: (a) considering that OEC isolation and expansion takes at least 6 weeks, there is a narrow window of opportunity for treatment of patients with acute ischemic conditions; (b) patient's clinical background and age can alter OEC functionality and numbers, for example, it has been reported that 
diabetes reduces the frequency of EPCs circulating in blood [18], and that they exhibit premature senescence $[19,20]$ thereby limiting cell numbers for therapy; (c) isolation efficiency for OECs from PB varies depending on the donor and the amount of blood. In our hands, starting with $40 \mathrm{~mL}$ of venous blood, isolation efficiency for OECs is around 30\%, so currently it is not possible to isolate EPCs from every patient. An attractive alternative to PB-OEC-based autologous therapies is the use of umbilical CB-derived OECs for allogeneic therapies. Umbilical $\mathrm{CB}$ has the advantage of being readily available, CB-OEC isolation efficiency is greater than $90 \%$, and our evidence shows that they can be expanded more rapidly than PB-OECs. Moreover, we have previously reported that $\mathrm{CB}-\mathrm{OEC}$ s can be frozen and stored in cryobanks without alterations to their growth potential, phenotype, and karyotype [11]. The immunocompatibility issue can be addressed by creating cell banks with detailed characterization of human leukocyte antigen (HLA) haplotypes to match population requirements. This methodology is currently being applied to stem cell banking of human embryonic stem cells in China [21] and human iPS cells in Japan [22]. Another approach frequently used in clinical trials for EPC isolation is the positive selection of CD34-expressing cells from $\mathrm{PB}$ or bone marrow by using a cell sorting approach. These CD34+ cells, although shown to be beneficial in promoting revascularization of ischemic hearts [17], contain only a fraction of "EPCs" as they are mainly composed of HSCs and mature endothelial cells. The search for a reliable marker to identify EPCs has proven extremely difficult and, to-date, cell culture methodologies represent a viable alternative to isolate highly homogeneous EPC populations such as OECs that can be ex vivo expanded and further functionally characterized $[6,11]$.

Cell culture is commonly associated with two types of senescence: replicative senescence and stress-induced premature senescence. While the former is directly dependent on telomere erosion, the latter is triggered by oxidative stress and DNA damage in the absence of significant telomere shortening. Our data on OEC expansion ex vivo shows cell proliferation with resultant telomere shortening and characterizes OEC senescence as being mainly replicative in nature. However, we cannot fully exclude the contribution of exogenous "stress factors" intrinsic to ex vivo culture conditions, indeed it has been reported that OECs undergo premature senescence without telomere shortening upon exposure to TNF- $\alpha$ [23]. In addition, the "chronological age" of OECs at the time of isolation might also play a role alongside the "replicative age" to determine the Hayflick limit as it was demonstrated in fibroblast strains that cells with higher replicative capacity had longer telomeres [24].

One important finding in this study was that senescence of OECs, due to ex vivo expansion, significantly impairs their functionality in vitro and diminishes their therapeutic potential in vivo. Therefore, the nature of OEC expansion and potential for senescence-related changes need to be taken into account when testing OECs in clinical trials.

Another interesting finding from this study is the identification of inflammatory cytokines as a major component of the OEC senescence program. This is in agreement with emerging observations highlighting that inflammatory proteins are induced in senescent cells [25]. In fact, cellular senescence now includes SASP as a novel hallmark [26]. Here we have characterized the SASP in senescent OECs and identified the cytokines IL8, IL6, IL $1 \alpha$, and IL1 $\beta$ as major components. These data clearly demonstrate that senescence-related changes in OECs go beyond simple growth arrest and actually involve a dynamic process whereby cells remain metabol- ically active and increase inflammatory cytokine production and secretion. This phenomenon has implications for the therapeutic use of OECs because these cytokines can alter tissue microenvironment conditions and affect cell engraftment, survival, and ultimately clinical outcomes. In keeping with our findings, it has been suggested that IL8 secreted by senescent cells fosters cancer progression by promoting both angiogenesis and tumor growth [27].

Among the novel findings of this study is the identification of IL8 as a major positive modulator of OEC senescence. We have shown that depletion of IL8 by shRNA in OECs leads to a significant delay in senescence, an increase in proliferation rates, and restored vasoreparative function. These data are in agreement with previous results demonstrating that knocking down the chemokine receptor CXCR2 (IL8RB) alleviates replicative and oncogene-induced senescence in fibroblasts by diminishing the DNA damage response [28]. Furthermore, a correlation between increased IL8 expression and $\mathrm{P} 16^{\mathrm{INK} 4 \mathrm{~A}}$ immunopositivity has been reported in growtharrested cells in human colorectal adenomas [25]. These findings alongside ours indicate an unexpected role for secreted inflammatory cytokines as autocrine regulators of senescence. Although a mechanistic model has been suggested where senescence-associated DNA damage triggers secretion of inflammatory cytokines which operate as positive-feedback loops by enhancing genes related to inflammation and cell cycle arrest [29], further studies are warranted to dissect respective molecular mechanisms. It is important to note that IL8 depletion in OECs did not fully bypass cellular senescence and this still occurred in IL8-knocked-down OECs, albeit at a significantly delayed time point. This suggests an enhancing and supporting role for IL8 in the senescence process, while indicating that $\mathrm{p} 53$ and $\mathrm{pRb}$ remain the critical components. Nevertheless, our results further advocate a prominent and clinically relevant role for regulation of IL8 and related signaling in OEC senescence, which could be a reasonable target to delay OEC senescence, without inducing uncontrolled cellular growth as has been described for other strategies targeting telomerase expression.

Identification of IL8 as one of the main components in the OEC secretome is in agreement with other reports [3033]. It has been shown that OECs secrete significantly more IL8 than human umbilical vein endothelial cells (HUVECs) or coronary artery endothelial cells [30]. Furthermore, IL8 secretion in OECs can be modulated by PAR-1 activation [32]. In this study, we demonstrate a significant increase of IL8 expression, production, and secretion that relates to cellular passage and the ex vivo expansion process of OECs. Furthermore, as IL8 levels increase during OEC expansion and relate to senescence establishment, this cytokine could potentially be used as a reliable ageing biomarker for OECs in vitro. As OEC cell number expansion is associated with impairment in cellular functionality, there is a clear need for senescence and functional screenings when OECs are expanded ex vivo. Therefore, it is also clinically relevant to highlight that our data shows evidence that IL8 levels in OEC conditioned media are a consistent and reliable indicator of their passage history and relate to functionality and senescence. While early passage OECs have IL8 levels $<50 \mathrm{pg} / \mathrm{ml}$, senescent OECs have levels ranging from 150 to $1,200 \mathrm{pg} / \mathrm{mL}$. These concentrations are very similar to the clinical parameters described for IL8 levels in human plasma when used as a biomarker for inflammation/infection [34].

Although we have evidence to indicate that IL8 acts as a provascular repair factor in the ischemic retina [35], the direct effects of IL8 on endothelial cells or OECs have not 
been clearly described. Evidence shows that while HUVECs respond to IL8 with a significant increase in their proliferation rate, human dermal microvascular endothelial cells failed to respond [36]. It is also important to highlight that, in the case of HUVECs, IL8-driven proliferation requires high IL8 concentrations (1-100 ng/mL). So far, only one report describes the direct effect of IL8 in OECs, and results indicate that, similar to HUVECs, very high doses of IL8 $(200 \mathrm{ng} / \mathrm{mL})$ were needed in order to induce a proangiogenic effect [37]; in addition, IL8 stimulation of OEC migratory capacity, although significant, was minor (sixfold lower) when compared to VEGF treatment. Our study has used IL8 concentrations ranging from 1 to $100 \mathrm{ng} / \mathrm{mL}$, and results indicate that recombinant human IL8 does not promote OEC proliferation or migration at these doses. However, OEC tube forming capacity was significantly enhanced by $1 \mathrm{ng} /$ mL IL8, but only in lower passage cells (up to passage P25) as higher passage OECs showed no angiogenic response when exposed to IL8. Our results underscore the complexity of the angiogenic process, and when dissected in its components, IL8 showed no effect upon OECs proliferation and migration; however, tubulogenesis in low-passage OECs was clearly enhanced by low concentrations of IL8. In summary, a proangiogenic role for exogenous IL8 in these highly proliferative OECs seems minor, while its intrinsic role as senescence facilitator is major and strongly supported by our data.

\section{REFERENCES}

1 Tongers J, Losordo DW, Landmesser U. Stem and progenitor cellbased therapy in ischaemic heart disease: Promise, uncertainties, and challenges. Eur Heart J 2011;32:1197-1206.

2 Fadini GP, Agostini C, Avogaro A. Autologous stem cell therapy for peripheral arterial disease meta-analysis and systematic review of the literature. Atherosclerosis 2010;209:10-17.

3 Stitt AW, O'Neill CL, O'Doherty MT et al. Vascular stem cells and ischaemic retinopathies. Progr Retinal Eye Res 2011;30:149-166.

4 Bernstein ID, Delaney C. Engineering stem cell expansion. Cell Stem cell 2012;10:113-114.

5 Yoder MC, Mead LE, Prater D et al. Redefining endothelial progenitor cells via clonal analysis and hematopoietic stem/progenitor cell principals. Blood 2007;109:1801-1809.

6 Reinisch A, Hofmann NA, Obenauf AC et al. Humanized large-scale expanded endothelial colony-forming cells function in vitro and in vivo. Blood 2009;113:6716-6725.

7 Wagner W, Bork S, Horn P et al. Aging and replicative senescence have related effects on human stem and progenitor cells. Plos One 2009; 4:e5846.

8 Chambers SM, Shaw CA, Gatza C et al. Aging hematopoietic stem cells decline in function and exhibit epigenetic dysregulation. Plos Biol 2007;5:e201.

9 Schallmoser K, Bartmann C, Rohde E et al. Replicative senescenceassociated gene expression changes in mesenchymal stromal cells are similar under different culture conditions. Haematologica 2010;95: 867-874.

10 Medina RJ, O'Neill CL, Sweeney M et al. Molecular analysis of endothelial progenitor cell (EPC) subtypes reveals two distinct cell populations with different identities. BMC Med Genom 2010;3:18.

11 Medina R, O'Neill CL, Humphreys MW et al. Outgrowth endothelial cells: Characterisation and their potential for reversing ischaemic retinopathy. Investig Ophthalmol Visual Sci 2010;51:5906-5913.

12 Kurz DJ, Decary S, Hong Y et al. Senescence-associated (beta)-galactosidase reflects an increase in lysosomal mass during replicative ageing of human endothelial cells. J Cell Sci 2000;113(pt 20):3613-3622.

13 Coppe JP, Patil CK, Rodier F et al. Senescence-associated secretory phenotypes reveal cell-nonautonomous functions of oncogenic RAS and the p53 tumor suppressor. Plos Biol 2008;6:2853-2868.

14 Kuilman T, Peeper DS. Senescence-messaging secretome: SMS-ing cellular stress. Nat Rev 2009;9:81-94.

15 Dubois C, Liu X, Claus P et al. Differential effects of progenitor cell populations on left ventricular remodeling and myocardial neovascula-

\section{Conclusions}

In conclusion, this work reveals that ex vivo expansion of human OECs ultimately leads to replicative senescence linked to cellular dysfunction. An inflammatory cytokine component was unequivocally identified as part of the OEC senescence program. Silencing IL8 expression in OECs resulted in delay of senescence and extension of OECs ex vivo lifespan which could have benefits in the context of vasoreparative or tissue engineering cell therapy.

\section{ACKNOWLEDGMenTS}

We thank Lynsey-Dawn Allen for constant technical support and assistance. This work was funded by grants from Fight for Sight UK, the Medical Research Council UK, the Sir Jules Thorn Trust, Royal Society, and Juvenile Diabetes Research Foundation.

\section{Disclosure of Potential CONFLICTS OF INTEREST}

The authors indicate no potential conflicts of interest.

rization after myocardial infarction. J Am College Cardiol 2010;55: 2232-2243.

16 Prockop DJ, Keating A. Relearning the lessons of genomic stability of human cells during expansion in culture: Implications for clinical research. Stem Cells 2012;30:1051-1052.

17 Sekiguchi H, Ii M, Losordo DW. The relative potency and safety of endothelial progenitor cells and unselected mononuclear cells for recovery from myocardial infarction and ischemia. J Cell Physiol 2009; 219:235-242.

18 Fadini GP, Sartore S, Albiero $M$ et al. Number and function of endothelial progenitor cells as a marker of severity for diabetic vasculopathy. Arterioscler Thromb Vasc Biol 2006;26:2140-2146.

19 Ingram DA, Lien IZ, Mead LE et al. In vitro hyperglycemia or a diabetic intrauterine environment reduces neonatal endothelial colonyforming cell numbers and function. Diabetes 2008;57:724-731.

20 Tepper OM, Galiano RD, Capla JM et al. Human endothelial progenitor cells from type II diabetics exhibit impaired proliferation, adhesion, and incorporation into vascular structures. Circulation 2002;106: 2781-2786.

21 Lin G, Xie Y, Ouyang Q et al. HLA-matching potential of an established human embryonic stem cell bank in China. Cell Stem cell 2009;5:461-465.

22 Okita K, Matsumura Y, Sato Y et al. A more efficient method to generate integration-free human iPS cells. Nat Methods 2011;8:409-412.

23 Zhang Y, Herbert BS, Rajashekhar G et al. Premature senescence of highly proliferative endothelial progenitor cells is induced by tumor necrosis factor-alpha via the p38 mitogen-activated protein kinase pathway. FASEB J 2009;23:1358-1365.

24 Dekker P, de Lange MJ, Dirks RW et al. Relation between maximum replicative capacity and oxidative stress-induced responses in human skin fibroblasts in vitro. J Gerontol 2011;66:45-50.

25 Kuilman T, Michaloglou C, Vredeveld LC et al. Oncogene-induced senescence relayed by an interleukin-dependent inflammatory network. Cell 2008;133:1019-1031.

26 Kuilman T, Michaloglou C, Mooi WJ et al. The essence of senescence. Genes Dev 2010;24:2463-2479.

27 Sparmann A, Bar-Sagi D. Ras-induced interleukin-8 expression plays a critical role in tumor growth and angiogenesis. Cancer Cell 2004;6: $447-458$.

28 Acosta JC, O'Loghlen A, Banito A et al. Chemokine signaling via the CXCR2 receptor reinforces senescence. Cell 2008;133:1006-1018.

29 Bartek J, Hodny Z, Lukas J. Cytokine loops driving senescence. Nat Cell Biol 2008;10:887-889.

$30 \mathrm{He}$ T, Peterson TE, Katusic ZS. Paracrine mitogenic effect of human endothelial progenitor cells: Role of interleukin-8. Am J Physiol 2005; 289:H968-H972. 
31 Yoon CH, Hur J, Park KW et al. Synergistic neovascularization by mixed transplantation of early endothelial progenitor cells and late outgrowth endothelial cells: The role of angiogenic cytokines and matrix metalloproteinases. Circulation 2005;112: $1618-1627$.

32 Smadja DM, Bieche I, Susen $\mathrm{S}$ et al. Interleukin 8 is differently expressed and modulated by PAR-1 activation in early and late endothelial progenitor cells. J Cell Mol Med 2009;13:2534-2546.

33 Hermansen SE, Lund T, Kalstad T et al. Adrenomedullin augments the angiogenic potential of late outgrowth endothelial progenitor cells. Am J Physiol Cell Physiol 2011;300:C783-C791.
34 Weitkamp JH, Reinsberg J, Bartmann P. Interleukin-8 (IL-8) preferable to IL-6 as a marker for clinical infection. Clin Diagn Lab Immunol 2002;9:1401.

35 Medina RJ, O'Neill CL, O'Doherty TM et al. Myeloid angiogenic cells act as alternative M2 macrophages and modulate angiogenesis through interleukin-8. Mol Med 2011;17:1045-1055.

$36 \mathrm{Li}$ A, Dubey S, Varney ML et al. IL-8 directly enhanced endothelial cell survival, proliferation, and matrix metalloproteinases production and regulated angiogenesis. J Immunol 2003;170:3369-3376.

37 Kimura T, Kohno H, Matsuoka $\mathrm{Y}$ et al. CXCL8 enhances the angiogenic activity of umbilical cord blood-derived outgrowth endothelial cells in vitro. Cell Biol Int 2011;35:201-208.

See www.StemCells.com for supporting information available online. 